\title{
Research on the Integrated Development of Intelligent Elderly Care Service in Beijing, Tianjin and Hebei
}

\author{
Bing-Rong LI \\ Business management research center of TUFE,Tianjin University of Finance and Economics, \\ Tianjin,China \\ Email:libingrong@tjufe.edu.cn \\ Corresponding author:Bingrong Li,13207595791
}

\begin{abstract}
In the context of sharp increase in aging population, both the Central Party Committee and the government have attached great importance to the elderly care issue. What's more, with the rapid development of science and technology, intelligent elderly care system is becoming more popular in today's world. This paper starts with an analysis of the background and current situation of the intelligent home care service in Beijing, Tianjin and Hebei. Based on the analysis, it points out the problems existing in the integration and development of this system -from the perspective of better integrating resources in the Beijing-Tianjin-Hebei region and offers corresponding suggestions to help this system move forward.
\end{abstract}

\section{Background of the Integrated Elderly Care Service}

The 13th Five-Year Plan represents a decisive stage in building a moderately prosperous society in all respects, and it is also an important strategic window period for China's reform in aging problem and construction of the elderly care system. In this crucial period, it is necessary to promote elderly care service for the aging population, especially in the field of intelligent home care service to speed up the coordinated development in Beijing, Tianjin and Hebei. On this very note, it makes sense to explore new ways and provide new opportunities by the advanced development of science and technology, networks and geopolitics in the three places.

According to the statistics of 2016, the aged population over 60 in Beijing accounts for $25.5 \%$ of the permanent residents, 23.35\% in Tianjin and 17.06\% in Hebei. According to the international standard, they have already entered an aging society. Recent years have witnessed a rapid increase in aging population. To combat this, one effective method is to push forward smart elderly care service.

\section{Analysis of the Current Development in Integrated Elderly Care Service}

On February 16, 2017, the Ministry of Industry and Information Technology, the Ministry of Civil Affairs, and the National Health and Family Planning Commission jointly issued the Action Plan for the Development of a Smart, Healthy, and Aged Industry (2017-2020). It is proposed that by 2020, a smart, healthy elderly care system which covers the entire life cycle will be basically formed, and a series of relevant brands will be created. In addition, the home care services such as health management and home-based elderly cares will be basically popularized, and the quality and efficiency are to be improved significantly.

\subsection{Analysis on the Commonalities and Problems}

With the development of smart products and technologies, they are gradually being applied to the elderly care service industry. For most old-age care institutions, they do not have a complete intelligent old-age care system which is equipped with complete data collection, processing, and early warning, so they have to rely on smart products to carry out daily monitoring of various indicators. The intelligent elderly care services launched in Beijing, Tianjin and Hebei are only at the pilot stage and have not been widely used. However, the local governments have listed the smart elderly care as their priority to promote elderly care service. 


\subsection{Commonalities of Intelligent Elderly Care Service}

\subsubsection{Home-oriented Intelligence Elderly Care Service}

Affected by past generations, residents in these regions tend to favor home-based care. As a response, smart elderly care services need to lay emphasis on how to send their products and services to households in need.

\subsubsection{Government-led Policies}

The local governments have been strongly supporting the development of the elderly care service industry as they attach importance to the application of smart technology and formulate many policies in the elderly care service industry, which are of great benefits to the elderly care service. The integration of Beijing, Tianjin and Hebei makes it much easier for the governments to explore new elderly care models jointly.

\subsubsection{Commonalities in the Market}

The residents of the three regions hold generally common views in elderly care, which is mainly affected by income and service level. Therefore, it makes sense to start from changing their perspectives, so as to expand new markets.

\subsection{Problems}

\subsubsection{Low Efficiency of the Traditional Elderly Care Industry}

Most of the elderly care institutions in the Beijing-Tianjin-Hebei region are in the red and have to rely on government financial subsidies. Given this, traditional elderly care institutions are calling for new technologies and development concepts.

\subsubsection{Inactive Elderly Care Model Led by the Government}

This, to some extent, hampers its development. In this connection, local governments in the three places must further embrace the market and increase market vitality and resource allocation efficiency.

\subsubsection{Less Marketization of Smart Elderly Care Technology}

The relevant technologies and systems for intelligent old-age care have achieved preliminary results, but lack of a technology transformation platform makes it hard to bring economic benefits.

\section{Problems that Desperately Need to be Solved in the Integration of Intelligent Elderly Care Service}

Focusing on intelligent old-age care, this paper makes an analysis on the basic elements, and believes that greater efforts should be made to integrate the development of the elderly care service

\subsection{Bringing in Research Outcomes Achieved by Local Scientific Research Institutes}

The three local governments need an industry-study-research cooperative mechanism to promote exchanges and cooperation among research institutions in the three places, including universities, research institutes, and smart elderly care-related companies, and strengthen the research on advanced technologies.

Establishing a transformation mechanism for achievements, according to the Beijing-Tianjin-Hebei Intelligent Cooperation Memorandum of Understanding, requires local departments to jointly build a pilot platform ,introduce the latest intelligent elderly care systems and models, establish pilot sites, and gradually expand their operations.

Promoting training of intelligent R\&D personnel and cultivation of professionals ,especially those who are specialized in computers and informatization can also at play in driving innovation. Increasing investment to attract more researchers including foreign professionals to carry out research will definitely be helpful in bringing in new ideas or views for domestic research. 


\subsection{Applying Technological Achievements both at Home and Abroad}

Firstly, elderly-care institutions need to learn experience from successful cases at home and abroad in an attempt to introduce more advanced intelligent elderly care technology or equipment and seek to develop more intelligent devices such as wearable devices and interactive robots to realize real-time and comprehensive monitoring and services.

Secondly ,it also helps to learn from the foreign models in Europe and the United States and do some research based on personal practices, such as the gap in income infrastructure and explore appropriate methods that are suitable for the current situation in Beijing, Tianjin and Hebei. In the whole process, they are required to gradually integrate intelligent technology and realize intelligent elderly care service.

\subsection{Attracting More Private Investment}

It works to lower the threshold for the elderly care market so that more private funds can be attracted. A market full of competition and dynamism plays an irreplaceable role in motivating related industries to generate more intelligent elderly care technologies and models. Besides, local governments need to issue more policies as support especially in areas such as land use and capital raising, so as to give preferential treatment to the social pension funds and attract more investment . Further improving the profit model will add to the attractiveness of intelligent elderly care service and the increase in profits will inevitably lead to more social capital.

\subsection{Integrating High-Quality Medical Resources}

The old are generally have higher requirements for medical resources and health services. Residents in Beijing, Tianjin, and Hebei are seeking for quality care services, especially for quality medical resources and health-care resources in consequence of their increasing income level.

In an effort to realize the healthy development of the elderly care industry and sharpen its competitive edge nationwide, Beijing, Tianjin and Hebei are required to gradually establish a medical cooperation mechanism so as to promote the integration of medical care and health care and integrate medical and service resources, realizing resource sharing, technology sharing, and platform sharing. What's more, the elderly care industry in Hebei should enjoy support from high-quality hospitals in Beijing and Tianjin by providing high-quality medical services.

\subsection{Building a Platform to Achieve Resource Integration}

To offer timely and effectively service to the elderly and meet the increasing diversified needs, on one hand, it is necessary to establish an intelligent data-collecting platform which can form a data analysis and feedback model by collecting data of different types of elderly people in the three regions. This platform is also able to integrate data from other platforms of different institutions and provide those institutions with feedback on the needs of the aged.

On the other hand, an intelligent resource-collecting platform covering Beijing, Tianjin, and Hebei is needed to make good use of resources of the three regions and then meet the needs of the elderly including food, shelter, clothing, transportation, entertainment and so on.

\section{Feasible Suggestions for the Integration of the Intelligent Elderly Care Service}

Based on the above research, the following countermeasures are proposed:

\subsection{Increasing Policy Support and Perfecting Rules and Regulations}

\subsubsection{Strengthening Governments' Coordination and Improving the Coordination Mechanism}

Based on a joint conference system, suitable rules and plans are asked to be issued and completed by three governments as soon as possible which includes the formulation and improvement of various policy systems, rational layout of institutions, capital raising and distribution, project approval and supervision, a clearly defined build process and access and exit mechanisms. 


\subsubsection{Enhancing Forceful Policy Support}

On the one hand, further support for intelligent zing the traditional public elderly care institutions is necessary. It will be very helpful to achieve marketization under the guidance of policies. On the other hand, local governments should playing a leading role in shoring up the development of private sectors and building brands. In an attempt to advance sustainable development, in-force policies are of great benefits in inducing innovation models of smart and health care service.

\subsubsection{Formulating a Unified Series of Service Standards}

Beijing, Tianjin and Hebei should formulate and publish as soon as possible a series of relevant standards and technical guidelines for intelligent old-age care services including standards for intelligent communities, institutions service and charging, admission of professional nursing staff standards, etc. Besides, scientific and advanced management system and methods will push the implementation of the service quality requirements, norms, systems, standards so as to satisfy the needs of the elderly population in big cities and enable senior citizens in relatively poor areas in Hebei to enjoy high-tech intelligent services.

\subsubsection{Strengthen the Supervision and Evaluation System}

While establishing standards, it is also necessary to set up a responsibility monitoring mechanism by which government departments can carry out strictly inspections over the community and institutions-the main bodies for investment and construction. The intelligent evaluation system and special evaluation agency set up by local governments will be conducive to the service assessment, supervision over the service quality and monitoring subsidy flows.

\subsection{Attracting More Private and Public Investment}

The economic development of the three places is unbalanced and there is still a large part of the elderly who do not have high incomes. On this very note, the government need to increase its subsidies to let every old person enjoy intelligent service. As a consequence, more old-age care service organizations will be created and more senior citizens can be served.

However, simply relying on government subsidies will undoubtedly become a burden for local governments, so the introduction of private capital will not only relieve government's financial pressure, but also promote intelligent elderly care industry and offer more jobs for the jobless.

\subsection{Improving Intelligent Systems and Strengthening Technological Cooperation}

The development of intelligent elderly care service is closely related to new technology. So, it is necessary to strengthen the cooperation in advanced technologies.

\subsubsection{Building an Integrated Intelligent Service Patform at a Professional and Technical Level}

The smart elderly care system is a platform that integrates internet of things, the Internet, and elderly care services. Through governmental open tender, setting up a unified elder-service network platform in Beijing, Tianjin and Hebei and classifying its content will surely secure the rapid flow of information and the sharing of information resources in three regions.

\subsubsection{Developing an Integrated Intelligent Service Terminal at the Data Mining Level}

In an effort to realize the healthy operation of smart old-age service mode, it works to dig information at the data collection terminal. Introducing competition into the market enable the terminal manufacturers to produce on demand so as to adapt to various functions developed by the intelligent elderly care network platform.

\subsubsection{Intensifying Technical Exchanges and Cooperation with Universities and Related Research Institutes}

The three regions need to establish long-term cooperation and exchanges with universities and research institutes which will boom advanced intelligent products. Pushing forward the 
industrialization of intelligent elderly care products will make it possible to reduce the cost of smart elderly care equipment, easing the economic burden of the old.

\subsection{Professional Training and Application of Smart Devices}

Talent integration is one of most important factors to promote intelligent elderly care service integration. So it is required to establish an all-round personnel training system in the three places and actively broaden the channels for the cultivation of talents, accelerating the development of professional management and building of service talents for the aged.

\subsubsection{Training and Assessment for Professional Care Staff}

There is a huge demand for service personnel in the three places. To advance intelligent old-age care, these institutions need not only to expand the number of nursing staff, but also to lay stress on the cultivation of compound talents with part-time nursing and information technology knowledge. Furthermore, they are required to improve assessment standards so as to improve staff's adaptability and proficiency in intelligent systems.

\subsubsection{Training for the Elderly to Use Smart Devices}

In spite of their limited ability to recognize and accept smart products, continuous training will work in helping the elderly overcome the actual and psychological obstacles and making smart terminal products more acceptable. At the same time, the training staff should be patient and timely solve various problems, so that the elderly can be proficient in using various intelligent terminal products.

\subsection{Breaking Geographical Restrictions and Promoting Long-Distance Elderly Care Service}

To break geographical restrictions and promote long-distance elderly care services, it is necessary to ensure the balanced allocation of old-age resources in the three places, which includes the infrastructure construction, the development of transportation integration and the sharing of medical resources. In addition, measures should be taken according to local conditions and based on their geographical advantages. For example, Beijing and Tianjin can utilize its technical advantages to build a high-end elderly care community. Hebei can build an ecological-based intelligent community and launch related products taking the advantage of its rich natural resources.

\section{Reference}

[1] Shiyang Liu, Ruifeng Li, Analysis on the Status Quo of Hebei's Endowment Service Industry[J] Management Review, 2015, 21, P19 22

[2] Wenguang Jia, Yingjun Du, "Internet + home care” Service Demand Status and Countermeasures[J] Shijiazhuang Vocational Technology College, 2017,04

[3] Yana Liu ,the Integration and Innovation of Endowment Mode Under the Coordinated Development of Beijing, Tianjin, and Hebei[J] China Administration, 2017, 07, P132 137

[4] Liying Yan, Pengliang Yan, Research on the Development Model of the Elderly Real Estate for the Qinhuangdao Under the Integration of Beijing, Tianjin and Hebei[J] Building Technology Development, 2016,10, P77 79

[5] Tianjin Economic Research Group, an Overview and Reference of Beijing-Tianjin-Hebei integration [J] Tianjin Economy, 2014.04, P22 29

[6] Yi Li, Mei Wang, Research on the Profit Model of Pension Industry-Taking the Pension Industry in Beijing, Tianjin, and Hebei as an Example[J]People's Forum, 2016,11, P226 228

[7] Peili Wang, Intelligent Home-Based Pension Model Based on Service Chain Theory_—Taking Tianjin as an Example[D] Master's Thesis, Tianjin Polytechnic University, 2015.12.06 
[8] Yumeng Liu, Design and Implementation of Intelligent Endowment System [D] Master of Engineering Dissertation, Beijing University of Technology, 2016.5

[9] Huimin Ma, The Status Quo and Development Prospects of Intelligent Elderly Care System: Taking Beijing and Tianjin as an Example[J], 2015, 06, P60

[10] Xuehua Meng, The current Situation and Countermeasures of the Standardization of China's Aged Care Service Industry[J] Quality Exploration, 2017.03, 56 61

[11] Yue Liu, The Status Quo, Problems and Countermeasures of the Development of China's Aged Care Service Industry[J] Science, Technology and Culture, 20

[12] Xingping Qian, The Current Situation and Countermeasures of Chinese Residents' Medical Support in the Background of Aging[J] China Rural Health Management, 2017.08, 881 883

[13] PeisonYan g, Thoughts on the Development Status and Coping Strategies of China's Aged Care Industry_-Based on the New Changes in China's Aging[J] Journal of Jilin Provincial Institute of Education, 2017.01, 135 138

[14] Lirui Dong, Cooperative Development of Endowment Service Industry in Beijing, Tianjin, Hebei Province[J] Manager, 2016.24,128

[15] Lili Wang, Xiaoqi Yang, Pengqi Dong, Analysis on the Status Quo of Urban Community Endowment Service Industry[J] Researches on Ageing, 2014.03,29 35

[16] Lihong Yan, Analysis and Research of Intelligent Endowment System Based on Internet of Things[J] Computer Knowledge and Technology, 2017.11, 247 248

[17] Yanan $\mathrm{Xu}$, Yue Zhao, Exploration of Intelligent Old-age Care in China[J] Modern Communication, 2017.06, 60 61

[18] Yan Jiao, Yunhong Li, Research on Innovation Model and Policy of Intelligent Old-age Care Industry in China[J] China Metallurgical Education, 2016.03, 30 34

[19] Junliang Zheng, China Community Public Intelligence Elderly Care Service Platform Construction [J] China Market, 2017.02, 142 145

[20] Peng Liu, On the Intelligent Elderly Care System The 31st China (Tianjin) 2017 IT, Network, Information Technology, Electronics, Instrumentation Innovation Conference, 245 247

[21] Pan Feng, Internet+ Community Aging: New Thinking on Intelligent Old-age Care[J] Learning and Practice, 2015.09, 99 105

[22] Jiansheng Wang, China's Modern Intelligent Old-age Care Will Gradually Become Intelligent [EB/OL] China Reform News, 2012.11.1

[23] Minli Wei, China will Build a Hundred Intelligent Old-age Experimental Base [EB/OL] Xinhuanet, 2012.10 